\title{
OSNR Penalty Imposed by Linear In-Band Crosstalk Caused by Interburst Residual Power in Multipoint-To-Point Networks
}

\author{
Bart Baekelandt, Student Member, IEEE, Cedric Mélange, Student Member, IEEE, \\ Johan Bauwelinck, Member, IEEE, Peter Ossieur, Member, IEEE, Tine De Ridder, Student Member, IEEE, \\ Xing-Zhi Qiu, Member, IEEE, and Jan Vandewege, Member, IEEE
}

\begin{abstract}
We present an analysis of linear in-band crosstalk in high split long reach wavelength/time-division-multiplexing passive optical networks (WDM-TDM PONs). In this letter, a mathematical model is deducted for the first time to calculate optical signal-to-noise ratio penalties due to in-band crosstalk in multipoint-to-point networks. The network performance can be perturbed by in-band crosstalk caused by power leakages from burst-mode optical network units (ONUs) in OFF-state. Our study results show that the leaked powers in upstream ONU transmitters can have an impact on the achievable split factor of WDM-TDM PONs. Furthermore, the performance limitations caused by aggregated interburst residual power are discussed.
\end{abstract}

Index Terms-Optical crosstalk, optical fiber communication, optical signal detection, optical transmitters, wavelength-division multiplexing (WDM).

\section{INTRODUCTION}

$\mathbf{T}$ HE presence of linear in-band crosstalk can severely impact the performance of wavelength-division-multiplexing (WDM) networks. It is mainly caused by nonperfect WDM crossconnects and, since the interferers are at the same wavelength as the transmitted signal, cannot be suppressed by additional filtering [1], [2].

In this letter, we describe another mechanism which can also cause in-band crosstalk. Fig. 1 depicts a high split long reach wavelength/time-division-multiplexing passive optical network (WDM-TDM PON). Several PON-like subnetworks, each with their own wavelength $\left(\lambda_{1}, \lambda_{2}, \ldots, \lambda_{n}\right.$, respectively), with a maximum of $10-\mathrm{km}$ fiber in the access portion and a maximum split factor up to 512, are aggregated onto a single feeder fiber in a dense WDM scheme with $90-\mathrm{km}$ feeder before the upstream signals are demultiplexed in front of an optical receiver at the optical line terminal (OLT), as shown in Fig. 1. All inactive optical network units (ONUs) must be switched OFF to avoid collisions upstream. The burst-mode ONU contains an externally modulated tunable laser with a built-in semiconductor optical amplifier (SOA).

Manuscript received October 23, 2007; revised January 8, 2008. This work was supported in part by the EU-funded PIEMAN project. The work of B. Baekelandt and C. Mélange was supported by Grants from the Institute for the Promotion of Innovation by Science and Technology in Flanders (IWT), Brussels. The work of P. Ossieur was supported by a Grant from the FWO.

The authors are with the Department of Information Technology (INTEC), Ghent University, B-9000 Gent, Belgium (e-mail: bart.baekelandt@intec. UGent.be).

Digital Object Identifier 10.1109/LPT.2008.918897

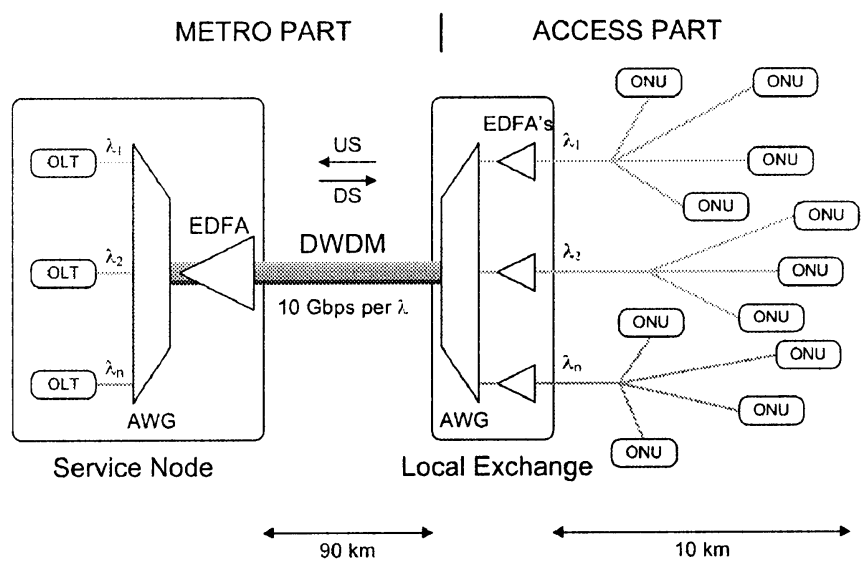

Fig. 1. Proposed network architecture (EDFA: erbium-doped fiber amplifier; DWDM: dense WDM; AWG: arrayed waveguide grating; US: upstream; DS: downstream).

In such a network, however, tight wavelength control of the ONU is of fundamental importance. This makes it difficult to switch OFF the tunable laser in between bursts on a nanosecond scale, as it disturbs the wavelength stabilization. One prefers instead to gate the integrated booster SOA postamplifier, located after the external modulator, in order to minimize the aggregated interburst residual power leaked from all inactive ONUs in the OFF-state. This residual power can introduce linear in-band crosstalk and degrade the network performance especially when the number of interfering ONUs exceeds 256 . This letter deduces a mathematical model to analyze the linear in-band crosstalk and focuses on the interplay between the crosstalk and the amplified spontaneous emission (ASE) noise caused by the gain-clamped erbium-doped fiber amplifiers in the network.

\section{Mathematical MODEL}

\section{A. In-Band Crosstalk}

Consider the signal from one PON subnetwork, impinging onto the photodiode in the OLT. The launched signal power of an active ONU is denoted $P_{\text {sig }}$. There will also be $N-1$ unmodulated interburst contributions $P_{\mathrm{OFF}, i}$ from all inactive ONUs in the network, $N$ being the split factor of the network, $i=$ $1, \ldots, N-1$. For this analysis, copolarization between transmitted signal $P_{\mathrm{sig}}$ and interferers is assumed, because changes in the state of polarization are slow; hence, the worst case of copolarization can persist for a considerable amount of time [3]. 
The photodetection current will contain, in addition to the usual ASE terms, a new crosstalk term $I_{X T}(t)$ caused by beating between the signal from the active ONU and the OFF-state signals

$$
I_{X T}(t)=2 R \sum_{i=1}^{N-1} \sqrt{P_{\mathrm{sig}} P_{\mathrm{OFF}, i}} \cdot \cos \left(\phi_{i}(t)-\phi_{s}(t)\right) .
$$

Without loss of generality, the responsivity $R$ will be assumed to be 1. $\phi(t)$ represents the phase noise of the corresponding source. The crosstalk term is essentially a sum of random terms and can be interpreted as intensity noise [4]. As the amount of interferers is typically relatively high, the central limit theorem can be used with good accuracy, leading to a Gaussian approach [5], [6]. The crosstalk variance $\sigma_{X T}^{2}$ is found as the mean square value of the crosstalk current

$$
\sigma_{X T}^{2}=\left\langle I_{X T}^{2}(t)\right\rangle=2 \cdot \sum_{i=1}^{N-1} P_{\mathrm{sig}} P_{\mathrm{OFF}, i} \cdot
$$

In an intensity modulated system with average received power $P_{\mathrm{REC}}$ and extinction ratio $\alpha$, the variances on one and zero levels are then given by

$$
\begin{aligned}
\sigma_{X T, 1}^{2} & =\frac{4}{\beta(1+\alpha)}(N-1) P_{\mathrm{REC}}^{2} \\
\sigma_{X T, 0}^{2} & =\frac{4 \alpha}{\beta(1+\alpha)}(N-1) P_{\mathrm{REC}}^{2}
\end{aligned}
$$

with

$$
\beta=\frac{P_{\mathrm{REC}}}{\left\langle P_{\mathrm{OFF}, i}\right\rangle} .
$$

In this equation, $\beta$ is the ratio of the received signal power to the average received interburst power and, for a given network configuration and ONU, is a constant. It is important to note that $\sigma_{X T}$ is directly proportional to $P_{\mathrm{REC}}$. This means that in a network dominated by in-band crosstalk, increasing the signal power will not improve network performance if the interburst residual power increases proportionally.

\section{B. OSNR Requirements}

In most practical systems, noise terms caused by ASE-ASE beating, crosstalk-crosstalk beating, and ASE-crosstalk beating are negligible. The dominant noise terms at the receiver are signal-crosstalk beat noise [given by (3)] and signal-ASE beat noise, given by

$$
\begin{aligned}
\sigma_{\mathrm{sig}-\mathrm{sp}, 1}^{2} & =4 \frac{2 P_{\mathrm{REC}}}{1+\alpha} N_{\mathrm{ASE}} \delta \nu_{\mathrm{el}} \\
\sigma_{\mathrm{sig}-\mathrm{sp}, 0}^{2} & =4 \frac{2 \alpha P_{\mathrm{REC}}}{1+\alpha} N_{\mathrm{ASE}} \delta \nu_{\mathrm{el}} .
\end{aligned}
$$

In these equations, $\delta \nu_{\mathrm{el}}$ is the electrical receiver bandwidth, and $N_{\mathrm{ASE}}$ is the ASE spectral density in one polarization. The optical signal-to-noise ratio (OSNR) at the receiver is given by OSNR $=\left(P_{\mathrm{REC}} / 2 N_{\mathrm{ASE}} B_{\mathrm{OSA}}\right)$, with $B_{\mathrm{OSA}}$ the reference bandwidth of the OSNR measurement, and substitution yields

$$
\begin{aligned}
\sigma_{\mathrm{sig}-\mathrm{sp}, 1}^{2} & =4 \frac{P_{\mathrm{REC}}^{2}}{1+\alpha} \frac{\delta \nu_{\mathrm{el}}}{B_{\mathrm{OSA}} \mathrm{OSNR}} \\
\sigma_{\mathrm{sig}-\mathrm{sp}, 0}^{2} & =4 \frac{\alpha P_{\mathrm{REC}}^{2}}{1+\alpha} \frac{\delta \nu_{\mathrm{el}}}{B_{\mathrm{OSA}} \mathrm{OSNR}} .
\end{aligned}
$$

The $Q$-factor at the receiver, which directly determines the biterror rate $(\mathrm{BER})$ through $\mathrm{BER}=(1 / 2) \operatorname{erfc}((Q) /(\sqrt{2}))$, is given by

$$
\begin{aligned}
Q & =\frac{I_{1}-I_{0}}{\sigma_{1}+\sigma_{0}} \\
& =\frac{2 \frac{1-\alpha}{1+\alpha} P_{\mathrm{REC}}}{\sqrt{\sigma_{X T, 1}^{2}+\sigma_{\mathrm{sig}-\mathrm{sp}, 1}^{2}}+\sqrt{\sigma_{X T, 0}^{2}+\sigma_{\mathrm{sig}-\mathrm{sp}, 0}^{2}}} \\
& =\frac{(1-\alpha)}{(1+\sqrt{\alpha}) \sqrt{1+\alpha} \sqrt{\frac{\delta \nu_{\mathrm{el}}}{B_{\mathrm{OSA} \mathrm{OSNR}}+\frac{(N-1)}{\beta}}}} .
\end{aligned}
$$

Solving for OSNR yields

$$
\mathrm{OSNR}=\frac{\delta \nu_{\mathrm{el}}}{B_{\mathrm{OSA}}} \frac{1}{\frac{(1-\alpha)^{2}}{(1+\sqrt{\alpha})^{2}(1+\alpha)} \frac{1}{Q^{2}}-\frac{N-1}{\beta}} .
$$

One can now define the crosstalk imposed OSNR penalty as the ratio of the OSNR requirement in a system with crosstalk to that in the same system without crosstalk

$$
\begin{aligned}
\delta_{\mathrm{osnr}} & =10 \log _{10} \frac{\frac{(1-\alpha)^{2}}{(1+\sqrt{\alpha})^{2}(1+\alpha)} \frac{1}{Q^{2}}}{\frac{(1-\alpha)^{2}}{(1+\sqrt{\alpha})^{2}(1+\alpha)} \frac{1}{Q^{2}}-\frac{N-1}{\beta}} \\
& =-10 \log _{10}\left(1-\frac{(1+\sqrt{\alpha})^{2}(1+\alpha)(N-1) Q^{2}}{(1-\alpha)^{2} \beta}\right) .
\end{aligned}
$$

When the signal and the interferers encounter exactly the same loss, $\beta$ is the ratio between the average launched signal power $P_{T X}$ and the interburst residual power $P_{\mathrm{OFF}, T}$. However, there exists differential losses in the access section. Denoting $L_{\mathrm{sig}}$ as the loss encountered by the signal burst and $L_{i}$ as the loss encountered by the contribution of ONU $i$, one can then split $\beta$ as

$$
\begin{aligned}
\beta & =\frac{P_{T X}}{L_{\mathrm{sig}}} \cdot \frac{1}{\left\langle\frac{P_{\mathrm{OFF}, T}}{L_{i}}\right\rangle} \\
& =\frac{P_{T X}}{P_{\mathrm{OFF}, T}} \cdot \frac{1}{\left\langle\frac{L_{\mathrm{sig}}}{L_{i}}\right\rangle}=\beta_{T X} \Delta L
\end{aligned}
$$

where the interburst suppression factor $\beta_{T X}$ is the ratio between the average launched signal power and the interburst residual power of all active ONUs, and $\Delta L$ represents the average difference in losses between the signal path and the interferers. Equation (9) then becomes

$$
\delta_{\mathrm{osnr}}=-10 \log _{10}\left(1-\frac{(1+\sqrt{\alpha})^{2}(1+\alpha)(N-1) Q^{2}}{(1-\alpha)^{2} \beta_{T X} \cdot \Delta L}\right) .
$$

Equation (11) calculates the OSNR penalty in an optically amplified multipoint-to-point network perturbed by in-band crosstalk, as a function of the ONU extinction ratio $\alpha$, the interburst suppression $\beta_{T X}$, the network split factor $N$, and the differential loss $\Delta L$ at a particular BER.

\section{RESULTS AND DISCUSSION}

Fig. 2 shows a plot of the OSNR requirement for a BER of $10^{-10}$ in a high split WDM-TDM PON where the signal 


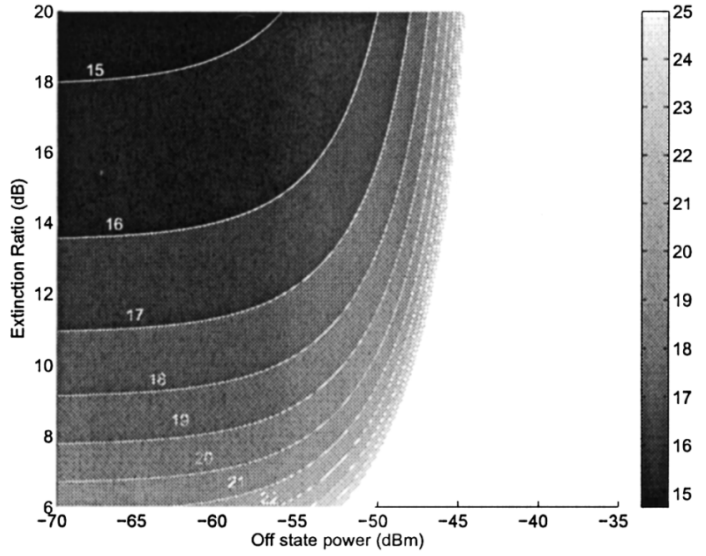

Fig. 2. OSNR requirement (decibels) for BER $=10^{-10}, \Delta L=5 \mathrm{~dB}, N=$ 512 .

from the active $\mathrm{ONU}$ encounters higher loss than the interferers $(\Delta L=5 \mathrm{~dB})$, which represents a bad network configuration for crosstalk. In the simulations, the average power of a signal burst is $5 \mathrm{dBm}$. For extremely low crosstalk, one would expect an OSNR requirement of around $17.5 \mathrm{~dB}$ for $\alpha=10 \mathrm{~dB}$. However, as the interburst power increases, the OSNR requirement increases before rapidly approaching infinity. Beyond this point, the BER floor imposed by crosstalk exceeds $10^{-10}$, and even a network without ASE noise would not attain the envisaged BER.

The upstream OSNR for an ONU employing the full network reach is around $21.5 \mathrm{~dB}$. Substracting an estimated burst mode penalty of $3 \mathrm{~dB}$, this becomes $18.5 \mathrm{~dB}$. In order to meet the OSNR requirement, the OFF-state power in this worst case of copolarization should be lower than $-54 \mathrm{dBm}$, which is a challenging design target, but possible to achieve using an SOA to gate the output of the ONU.

Fig. 3 shows the OSNR requirement and the OSNR penalty for $N=512$, in a case where forward-error correction (FEC) is employed, assuming a pre-FEC BER of $10^{-4}$. With a split factor of 512, extinction ratio of $10 \mathrm{~dB}$, and a worst-case differential loss of $10 \mathrm{~dB}$, the interburst residual output power should be lower than $-48.5 \mathrm{dBm}$ for an OSNR requirement of $18.5 \mathrm{~dB}$. FEC codes that yield a post-FEC BER of $10^{-10}$ have been demonstrated [7].

Decreasing the split factor will improve the situation twofold: first, because the number of interferers is lower, the OSNR penalty and hence the OSNR requirement will be lower. Second, the achievable OSNR will be higher, because losses in the access part will be lower. This means that a higher OSNR penalty can be tolerated.

\section{CONCLUSION}

In this letter, linear in-band crosstalk caused by interburst residual power in multipoint-to-point networks was introduced. Based on a Gaussian approximation, an analytical model was

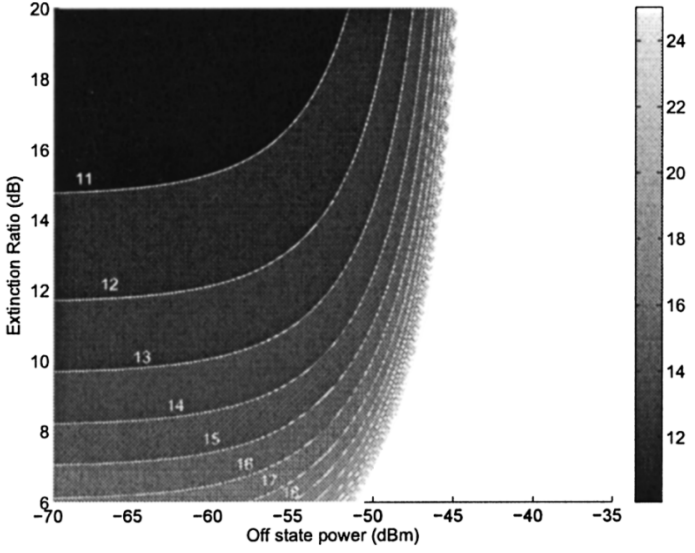

(a)

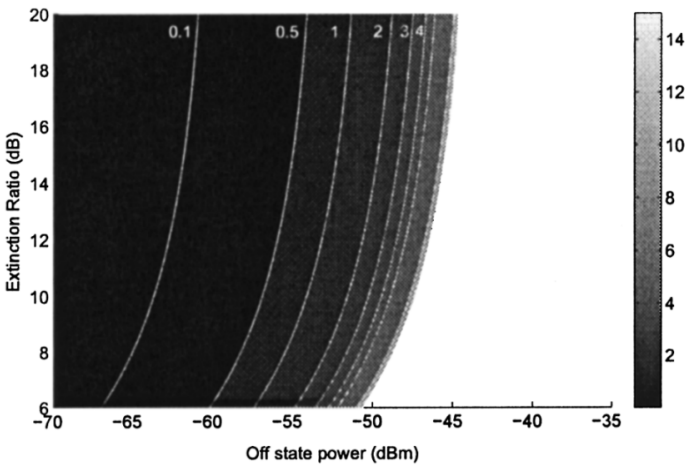

(b)

Fig. 3. (a) OSNR requirement with FEC at pre-FEC BER $=10^{-4}, \Delta L=$ $10 \mathrm{~dB}, N=512$. (b) OSNR penalty.

presented, which allows the calculation of OSNR requirements in networks perturbed by ASE noise and in-band crosstalk, as well as the OSNR penalties imposed by in-band crosstalk.

\section{REFERENCES}

[1] H. Takahashi, K. Oda, and H. Toba, "Impact of crosstalk in an arrayedwaveguide multiplexer on $N \times N$ optical interconnection," J. Lightw. Technol., vol. 14, no. 6, pp. 1097-1105, Jun. 1996.

[2] E. L. Goldstein and L. Eskildsen, "Scaling limitations in transparent optical networks due to low-level crosstalk," IEEE Photon. Technol. Lett., vol. 7, no. 1, pp. 93-94, Jan. 1995.

[3] I. T. Monroy and E. Tangdiongga, Crosstalk in WDM Communication Networks. Boston, MA: Kluwer, 2002.

[4] G. P. Agrawal, Fiber-Optic Communication Systems, 3rd ed. New York: Wiley, 2002.

[5] J. C. Attard, J. E. Mitchell, and C. J. Rasmussen, "Performance analysis of interferometric noise due to unequally powered interferers in optical networks," J. Lightw. Technol., vol. 23, no. 4, pp. 1692-1703, Apr. 2005.

[6] M. R. Jimenez, R. Passy, M. A. Grivet, and J. P. von der Weid, "Computation of power penalties due to intraband crosstalk in optical systems," IEEE Photon. Technol. Lett., vol. 15, no. 1, pp. 156-158, Jan. 2003.

[7] M. Rasztovits-Wiech and A. Stadler et al., "Realization of an XL-PON prototype," in Proc. BroadBand Eur. 2007, Antwerp, Belgium, Dec. 3-6, 2007, Paper We3B2, ISBN 9789076546094. 